\title{
Superficial temporal artery-to-middle cerebral artery bypass in combination with indirect revascularization in moyamoya patients $\leq 3$ years of age
}

\author{
Gyang Markus Bot, BM, BCh, ${ }^{1,3,4}$ Jan-Karl Burkhardt, MD, ${ }^{1}$ Nalin Gupta, MD, PhD, ${ }^{1,2}$ and \\ Michael T. Lawton, MD 4
}

\begin{abstract}
Departments of ${ }^{1}$ Neurological Surgery and ${ }^{2}$ Pediatrics, University of California, San Francisco, California; ${ }^{3}$ Neurosurgery Division, Department of Surgery, Jos University Teaching Hospital, Jos, Plateau State, Nigeria; and ${ }^{4}$ Department of Neurological Surgery, Barrow Neurological Institute, Phoenix, Arizona
\end{abstract}

\begin{abstract}
OBJECTIVE Revascularization is indicated in the management of moyamoya disease (MMD), with options that include direct and indirect techniques. Indirect bypass is popular in young children because the diminutive caliber of donors and recipients makes direct bypass difficult. The authors reviewed a series of patients treated with direct superficial temporal artery (STA)-to-middle cerebral artery (MCA) bypass in combination with encephalomyosynangiosis (EMS) in children 3 years or younger to demonstrate feasibility and safety.
\end{abstract}

METHODS A retrospective review of all surgeries for MMD over a 19-year period identified 11 procedures in 6 patients. Surgical results, angiographic outcomes, and clinical outcomes were analyzed.

\begin{abstract}
RESULTS Patients had a mean age of 22.4 months. The symptomatic hemisphere was revascularized first, and the contralateral hemisphere was revascularized on average 2.8 months later in 5 patients. All direct bypasses were patent postoperatively and remained patent at late follow-up (mean 4.1 years), with both STA and MCA diameters increasing significantly $(n=5, p<0.03$ ). At last follow-up (mean follow-up duration, 5.0 years), favorable outcomes (modified Rankin Scale scores $0-2$ ) were observed in 5 of the 6 patients $(83 \%)$, with 1 dependent patient remaining unchanged postoperatively.
\end{abstract}

CONCLUSIONS Direct STA-MCA bypass in combination with EMS for MMD is feasible and safe in patients 3 years or younger, based on favorable clinical and radiological outcomes in this patient cohort. Direct bypass should be considered when immediate revascularization is needed, without the biological delay associated with indirect bypass.

https://thejns.org/doi/abs/10.3171/2018.9.PEDS18224

KEYWORDS moyamoya disease; direct and indirect revascularization technique; STA-MCA bypass; long-term outcome; vascular disorders

$\mathrm{M}$ Oyamoya disease (MMD) is the most common cause of pediatric stroke with a prevalence of 3 per 100,000 patients in the Japanese population and an incidence of 0.086 per 100,000 persons in the US. ${ }^{11}$ MMD is approximately twice as common in females than in males and has a bimodal distribution-during childhood at age 5 years and during adulthood in the mid-40s. ${ }^{11}$ This chronic, progressive cerebrovascular arteriopathy is characterized by stenosis and subsequent occlusion of the distal internal carotid arteries, proximal anterior cerebral arteries, and proximal middle cerebral arteries, leading to the formation of fragile, collateral anastomoses and presen- tation with transient ischemic attacks (TIAs) or strokes. ${ }^{5,11}$ Revascularization surgery has proven to be effective in preventing further stroke.,12,17 Direct revascularization utilizes an extracranial-intracranial superficial temporal artery-to-middle cerebral artery (STA-MCA) bypass, ${ }^{13}$ while indirect revascularization elicits angiogenic connections with encephaloduroarteriosynangiosis (EDAS) or encephalomyosynangiosis (EMS) without direct anastomosis with suturing. ${ }^{5,13}$ Combinations of both indirect and direct revascularization are also performed..$^{2,10,13}$

Direct revascularization is the revascularization procedure of choice in our practice in older children and adults.

ABBREVIATIONS EDAS = encephaloduroarteriosynangiosis; EMS = encephalomyosynangiosis; $M C A=$ middle cerebral artery; MMD = moyamoya disease; mRS = modified Rankin Scale; STA = superficial temporal artery; TIA = transient ischemic attack.

SUBMITTED April 18, 2018. ACCEPTED September 7, 2018.

INCLUDE WHEN CITING Published online November 16, 2018; DOI: 10.3171/2018.9.PEDS18224. 
Many studies report a preference for indirect revascularization procedures in small children ${ }^{2,5}$ and in patients 3 years or younger, mainly due to the small caliber of donor and recipient vessels. ${ }^{2,15}$ However, it is known that MMD in young children progresses more rapidly than in older children and adults, with a higher risk of stroke and poor clinical outcome if untreated or delayed in treatment. ${ }^{5}$ Indirect revascularization does not augment flow to the hemisphere immediately, which leaves patients at risk for stroke perioperatively and during the time it takes for the angiogenesis response to the graft. This delay in flow augmentation may make direct bypass the better option because hemodynamics improve immediately and protect the brain from ischemia. ${ }^{13}$ In this study, we analyzed the outcomes and complications of pediatric moyamoya patients 3 years or younger treated with a direct STA-MCA bypass in combination with an indirect revascularization technique to demonstrate the feasibility of direct bypass in this population. This study is not intended to demonstrate superiority over more widely used indirect bypass techniques in this age group.

\section{Methods}

\section{Inclusion Criteria}

This study was approved by the institutional review board at the University of California, San Francisco, and performed in compliance with Health Insurance Probability and Accountability Act regulations. A retrospective review of all surgeries for MMD in patients 3 years or younger at the time of surgery treated by the senior author (M.T.L.) between January 1998 and September 2016 identified 6 patients with 11 procedures.

\section{Clinical and Radiological Data}

Clinical data, including age, sex, clinical symptoms, associated syndromes, and perioperative complications, as well as clinical outcome using the modified Rankin Scale (mRS), were collected. In addition, type of surgery and radiological data including the pre- and postoperative diameters of the STA and MCA, if available, and bypass patency were collected.

\section{Surgical Technique \\ Direct Revascularization}

In all cases, an end-to-side extracranial-intracranial low-flow STA-MCA bypass was performed. After harvest of both frontal and parietal STA branches (if available), the more prominent branch (single barrel) or both branches (double barrel) were chosen for the direct anastomosis. The STA was fish-mouthed and sutured with continuous 10-0 sutures to a cortical $\mathrm{M}_{4}$ MCA branch. Clamp time for the bypass ranged from 20 to 30 minutes. Bypass patency was confirmed intraoperatively with indocyanine green videoangiography. All patients were started on a daily aspirin regimen after the bypass procedure.

\section{Indirect Revascularization}

After completing the direct bypass, EMS was performed. After widely opening the arachnoid mater, the temporalis muscle was cleaved in half in its sagittal plane, and the inner portion was used as an indirect onlay graft on the cortex and sutured superiorly to the dural edge. After replacing the bone flap, the outer portion of muscle was reapproximated with the muscle cuff extracranially.

In patients who did not undergo a direct bypass, EDAS was performed. Here, the STA was harvested similar to that in the direct revascularization technique and sutured to the arachnoid with interrupted $10-0$ sutures without a direct bypass.

\section{Statistical Analysis}

Statistical analysis was performed using IBM SPSS (version 22, IBM Corp.), and figures were generated using Prism software (GraphPad). Continuous variables are presented as the mean with standard error of the mean or range. Comparisons between groups were performed using the Mann-Whitney U-test for continuous parameters and the chi-square test or the Fisher's exact test for categorical parameters. Statistical significance was established at the alpha level of $\mathrm{p}=0.05$.

\section{Results}

Overall, 11 procedures were completed in 6 patients 3 years or younger, with a mean age of 22.4 months (SD 9.5 months) at the time of surgery. All patients were symptomatic with stroke $(n=5)$ or TIA only $(n=1)$ (Table 1$)$.

Most patients $(83 \%, 5 / 6)$ received bilateral revascularization procedures, and the symptomatic hemisphere was treated first due to a symptomatic stroke or TIA. The contralateral hemisphere was treated prophylactically in 4 of 5 patients $(80 \%)$. A direct STA-MCA bypass in addition to an ipsilateral EMS was performed in 91\% (10/11) of the cases. The STA-MCA bypass anastomosis was performed as a single-barrel bypass in $90 \%$ of the cases (9/10), and as a double-barrel STA-MCA bypass in 1 case (Table 1). The mean time interval between surgeries was 2.8 months (0.2-8.6 months) in the 5 patients. One patient had a new stroke on the contralateral side during this interval between surgeries (Fig. 1).

One patient had already received previous bilateral indirect EDAS revascularizations at a different hospital and became symptomatic with a new left hemispheric stroke. This patient received an STA-MCA bypass, recovered well without new symptoms, and had a patent bypass 8.1 years after surgery. The right EDAS successfully augmented flow on that side; therefore, no additional surgery was needed.

Postoperative catheter angiography confirmed bypass patency in all 10 direct revascularizations and 11 anastomoses (10 single-barrel STA-MCA bypasses and 1 double-barrel STA-MCA bypass). The mean time for late follow-up imaging was 4.1 years (range $0.8-7.6$ years). Vessel diameter significantly increased at the last follow-up compared with the diameter on preoperative angiography in 5 available cases: the STA diameter increased from a mean diameter of $0.92 \mathrm{~mm}$ (range $0.57-1.09$ ) to $1.53 \mathrm{~mm}$ (range $0.97-1.87)(\mathrm{p}=0.03)$ and the MCA diameter increased from $0.67 \mathrm{~mm}$ (range $0-1.4$ ) to $1.49 \mathrm{~mm}$ (range 0.85-1.09) $(\mathrm{p}=0.03)$ (Fig. 2). 


\begin{tabular}{|c|c|c|c|c|c|c|c|c|c|c|c|c|c|}
\hline $\begin{array}{l}\text { Case } \\
\text { No. }\end{array}$ & $\begin{array}{l}\mathrm{Pt} \\
\text { No. }\end{array}$ & $\begin{array}{c}\text { Age } \\
\text { (mos), } \\
\text { Sex }\end{array}$ & $\begin{array}{l}\text { Suzuki } \\
\text { Stage }\end{array}$ & Indication for Op & Type of Op & $\begin{array}{c}\text { Side } \\
\text { of } \\
\text { Op }\end{array}$ & $\begin{array}{c}\text { Previous } \\
\text { Op } \\
\text { Same } \\
\text { Side }\end{array}$ & $\begin{array}{c}\text { Other } \\
\text { Diagnosis }\end{array}$ & $\begin{array}{l}\text { Postop } \\
\text { Stroke/Sz }\end{array}$ & $\begin{array}{l}\text { Other } \\
\text { Comps }\end{array}$ & $\begin{array}{l}\text { LFU } \\
\text { (yrs) }\end{array}$ & $\begin{array}{l}\text { Bypass } \\
\text { Patent } \\
\text { at LFU }\end{array}$ & $\begin{array}{c}\mathrm{mRS} \\
\text { Score } \\
\text { at } \\
\text { LFU }\end{array}$ \\
\hline 1 & 1 & $17, \mathrm{~F}$ & III & $\begin{array}{l}\text { Hemiparesis, } \\
\text { stroke on MRI }\end{array}$ & sSTA-MCA plus EMS & $\mathrm{Lt}$ & No & None & No & No & 2.6 & Yes & 2 \\
\hline 2 & 1 & $17, \mathrm{~F}$ & II & Asymp stroke & sSTA-MCA plus EMS & Rt & No & None & No & No & 2.5 & Yes & 2 \\
\hline 3 & 2 & $22, \mathrm{~F}$ & III & $\begin{array}{l}\text { Hemiparesis \& } \\
\text { stroke }\end{array}$ & sSTA-MCA plus EMS & $\mathrm{Lt}$ & No & None & No & $\begin{array}{l}\text { Wound } \\
\text { revision }\end{array}$ & 7.0 & Yes & 1 \\
\hline 4 & 2 & $30, \mathrm{~F}$ & I & No Sxs, preventive & sSTA-MCA plus EMS & Rt & No & None & No & No & 6.3 & Yes & 1 \\
\hline 5 & 3 & $16, M$ & IV & $\begin{array}{l}\text { New stroke, hemi- } \\
\text { paresis }\end{array}$ & sSTA-MCA plus EMS & $\mathrm{Lt}$ & EDAS & MOPD2 & No & No & 8.1 & Yes & 3 \\
\hline 6 & 4 & $36, \mathrm{M}$ & III & $\begin{array}{l}\text { TIA, no stroke on } \\
\text { MRI }\end{array}$ & sSTA-MCA plus EMS & Rt & No & None & No & No & 4.9 & Yes & 0 \\
\hline 7 & 4 & $36, \mathrm{M}$ & II & No Sxs, preventive & sSTA-MCA plus EMS & $\mathrm{Lt}$ & No & None & No & No & 4.9 & Yes & 0 \\
\hline 8 & 5 & $9, F$ & IV & $\begin{array}{l}\text { Sz, hemiparesis, } \\
\text { stroke on MRI }\end{array}$ & $\begin{array}{l}\text { dSTA-MCA plus } \\
\text { EMS }\end{array}$ & $\mathrm{Lt}$ & No & None & No & No & 8.1 & Yes & 2 \\
\hline 9 & 5 & $11, \mathrm{~F}$ & II & No Sxs, preventive & EDAS & Rt & No & None & No & No & 8.0 & NA & 2 \\
\hline 10 & 6 & $23, \mathrm{~F}$ & IV & TIA, stroke on MRI & sSTA-MCA plus EMS & Rt & No & AML & $\begin{array}{r}\text { New postop } \\
\text { stroke/Sz }\end{array}$ & No & 1.4 & Yes & 2 \\
\hline 11 & 6 & $27, \mathrm{~F}$ & III & $\begin{array}{l}\text { Hemiparesis, new } \\
\text { stroke }\end{array}$ & sSTA-MCA plus EMS & $\mathrm{Lt}$ & No & AML & No & No & 1.1 & Yes & 2 \\
\hline
\end{tabular}

AML = acute myelogenous leukemia; Asymp = asymptomatic; Comps = complications; dSTA-MCA = double-barrel STA-MCA; LFU = last follow-up; MOPD2 = Majewski osteodysplastic primordial dwarfism type II; NA = not applicable; $\mathrm{Pt}=$ patient; $\mathrm{sSTA}-\mathrm{MCA}=$ single-barrel STA-MCA; Sxs = symptoms; $\mathrm{Sz}=$ seizure .

One patient needed superficial surgical wound revision 1 month after bypass surgery but recovered well without neurological deficits (Table 1). Clinical outcomes were favorable in $91 \%(10 / 11)$ of the surgeries with 1 new postoperative symptomatic stroke in the anterior cerebral artery territory causing leg weakness and seizure 24 hours after surgery, but without deficits at the last follow-up. Favorable outcomes (mRS scores $0-2$ ) were observed in 83\% (5/6) of the patients; 1 patient had an mRS score of 3 , which was unchanged from the preoperative status. The mean followup duration was 5.0 years (range 1.1-8.1 years).

\section{Discussion}

MMD in young children is characterized by a fulminant clinical course with rapid progression and poor clinical outcome if untreated. ${ }^{1,67,15}$ Therefore, treatment with surgical revascularization is indicated for most symptomatic patients. Direct revascularization can be challenging due to the small arterial diameters of both donor and recipient, but offers immediate and reliable revascularization. This study documenting our experience with direct revascularization in pediatric moyamoya patients 3 years or younger demonstrates the feasibility of this bypass. This study does not demonstrate, and was not intended to demonstrate, superiority over more widely used indirect bypass techniques in this age group.

\section{Demographics and Clinical Presentation}

MMD was more than twice as common in females than males in our patient cohort, which is similar to the ratio described in the literature..$^{11}$ All of our patients were symptomatic with either stroke or TIA, which is also consistent with the literature. Kim et al. showed in 204 patients with pediatric MMD that $87 \%$ of the patients in the age group of 3 years or younger presented with symptomatic stroke. ${ }^{6}$ Jackson et al. reported TIAs in $94.7 \%$ of patients at presentation, stroke in $89.5 \%$, and seizures in $21.1 \% .^{5}$ These results are similar to our findings with stroke in 5 of 6 patients $(83.3 \%)$ and preoperative seizure in 1 of 6 patients $(16.7 \%)$.

\section{Revascularization Surgery}

Indirect revascularization surgery is the popular surgical technique in young MMD children due to the small vessel caliber and the technical difficulty of direct anastomosis. ${ }^{2,5,6} \mathrm{We}$ prefer direct revascularization because immediate blood flow augmentation protects the hemisphere at risk from further strokes. ${ }^{8}$ Indirect revascularization requires weeks to months for augmentation of blood flow. ${ }^{11}$ The combination of both direct and indirect techniques in MMD and moyamoya syndrome achieves immediate revascularization with the opportunity for further indirect anastomosis over time. ${ }^{3,9,14}$ We were able to show that direct STA-MCA bypass procedures were possible in most of our patients ( 10 of 11 procedures, $91 \%$ ), with the only indirect revascularization performed due to an absent STA on the asymptomatic hemisphere after successful direct STA-MCA bypass on the contralateral symptomatic side. The diminutive arterial diameters in this population and their more delicate tissues make the the vascular neurosurgeon's level of experience more important than in 

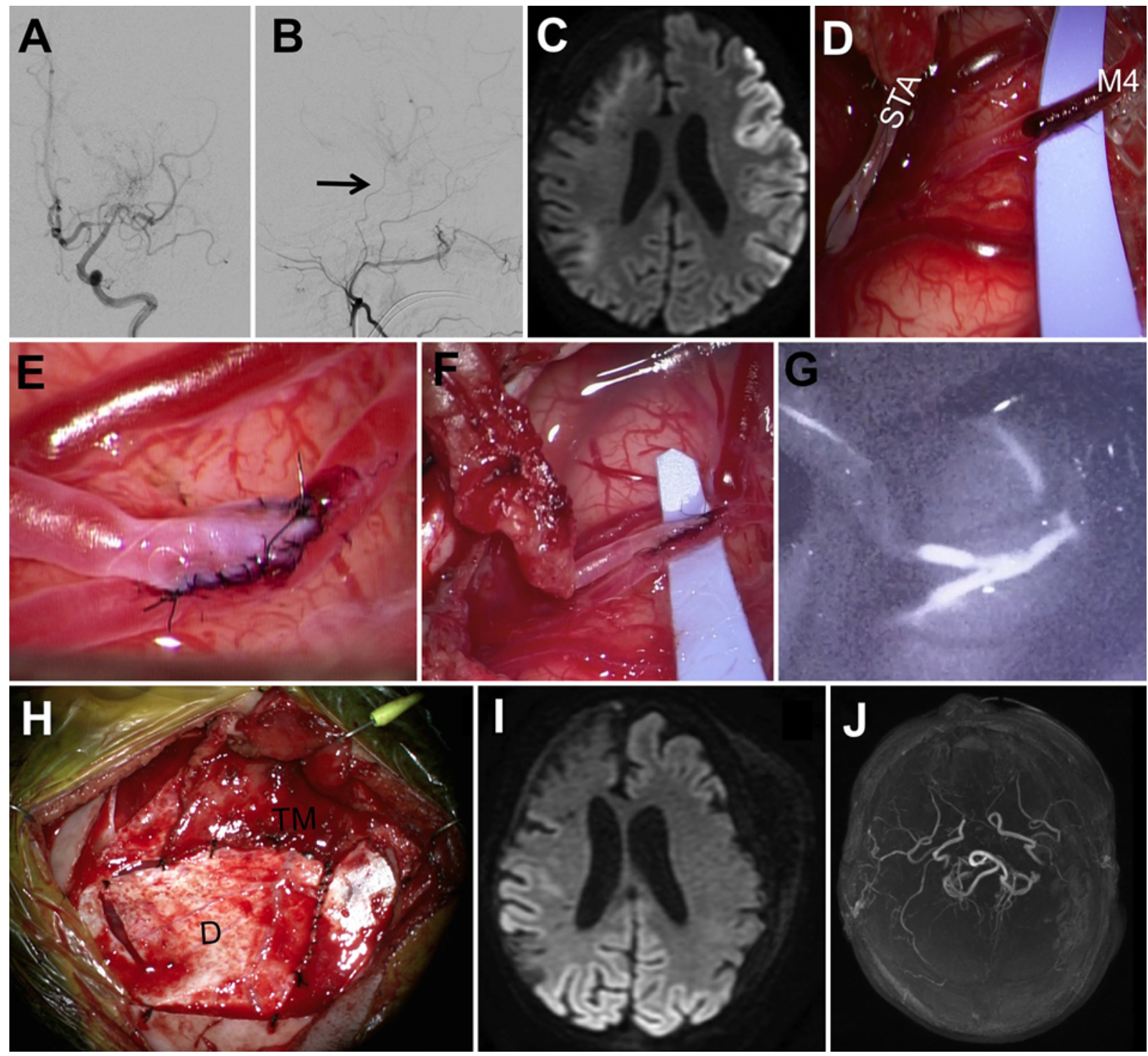

FIG. 1. Patient 6. This 23-month-old girl with Down syndrome and bilateral moyamoya syndrome received a right STA-MCA bypass after a right hemispheric stroke. Four months after surgery, the patient presented with a new stroke, this time involving the left hemisphere with a right arm weakness. A and B: Catheter angiography of the left internal carotid artery (A) showed the classic pattern of moyamoya syndrome, and an external carotid artery injection showed a small, but present STA (B, arrow). C: Ischemia in the left MCA territory was seen on MR imaging. After harvesting the STA, a mini pterional craniotomy was performed, and an $\mathrm{M}_{4}$ segment was selected as a recipient artery for the end-to-side anastomosis. D: The donor artery was fish-mouthed and approximated to the recipient with 2 interrupted 10-0 sutures. E: Running continuous sutures were sewn along both suture lines. F and G: After completion of the anastomosis, temporary clips were removed $(F)$ and indocyanine green videoangiography showed early filling of the $\mathrm{M}_{4}$ branch through the STA $(\mathrm{G})$. H: Before closure, EMS was performed as an indirect bypass (D = dura; TM = temporalis muscle). I and J: The patient recovered well, and postoperative MRI and MRA demonstrated recovery of the stroke on diffusion-weighted imaging (I) and patency of bilateral STA-MCA bypasses $(\mathrm{J})$. Figure is available in color online only.

older children or adults. In this series, all bypasses were performed by the senior author who had more than 10 years of experience performing these bypasses. The fishmouth arteriotomy in the donor STA and a long linear arteriotomy in the recipient are larger than in other patients (i.e., older children and adults) because these techniques increase the area of the anastomosis in these small vessels.

\section{Perioperative Complications}

Young children have a known higher risk of periopera- tive stroke. Kim et al. reported a $39 \%$ stroke risk in patients younger than 3 years, compared with $6 \%$ and $0.6 \%$ for children between the ages of 3 and 6 years and those older than 6 years, respectively. ${ }^{6}$ Our perioperative stroke risk was low, with only one patient experiencing a new symptomatic perioperative stroke which resolved at LFU. The low perioperative stroke risk may be due to the immediate revascularization of direct bypass without the biological delay associated with indirect revascularization. The risk of other complications of direct revasculariza- 

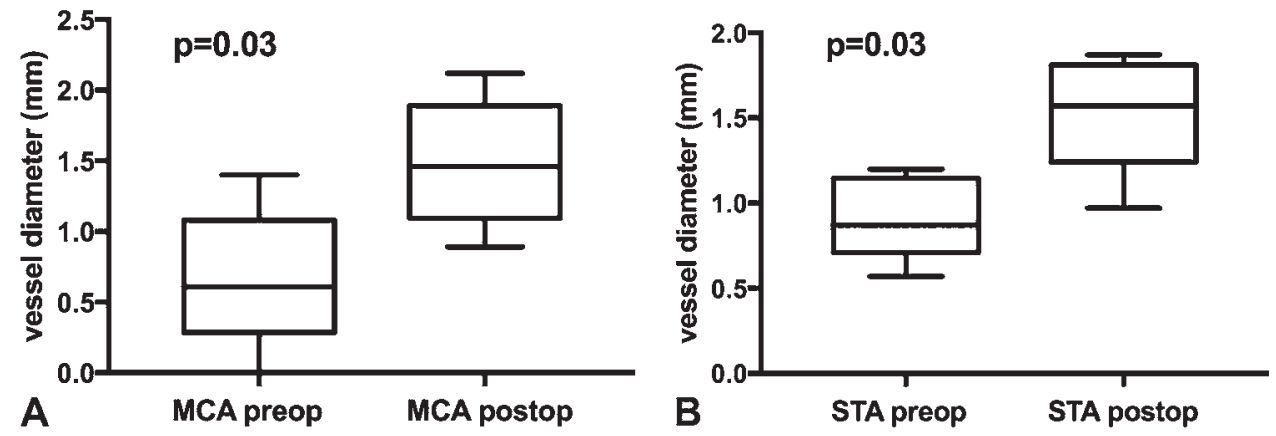

FIG. 2. Box plots showing MCA (A) and STA (B) vessel diameters before and after direct STA-MCA bypass procedures in 5 patients. Boxes represent the IQR of the median (lines within the boxes), and whiskers represent the entire range.

tion may be higher than indirect revascularization, including postoperative intracranial hemorrhage, asymptomatic MRI changes, bypass occlusion, and severe neurological deficits, ${ }^{16}$ but these complications were not observed in our cohort.

\section{Radiological and Clinical Outcome}

Radiological outcomes were favorable in our cohort with patency of all bypasses and only one patient with new documented ischemia on postoperative MRI. Both STA and MCA diameter significantly increased in size at last follow-up compared with before surgery $(p=0.03)$, suggesting robust revascularization and protection from future stroke. Robust bypasses are not expected to impact the progression of underlying disease, and the study by Jackson et al. showed radiological progression of the MMD in patients who had a pre- and postoperative angiography after indirect revascularization in children younger than 2 years, despite clinical improvement and a good outcome. ${ }^{5}$

Clinical outcomes were favorable in all of our patients, which contrasts with some previous studies. Kim et al. reported favorable outcome (mRS scores $0-2$ ) in 58\% in children younger than 3 years compared with $85 \%$ and $86 \%$ for those patients between 3-6 years and $>6$ years, respectively. ${ }^{6}$ Other studies of children younger than 2 years showed a favorable outcome in 68.4\%.5 Although lower than our results with direct revascularization, these outcomes with indirect revascularization still show a better outcome than the natural history of MMD, with a stroke rate of $65 \%-82 \% .5,8$

Direct bypass in patients within their first 3 years of life has only been reported by Rashad et al., who included children 2-16 years old in whom direct bypass was successful without perioperative stroke or hemorrhage..$^{10}$ Our favorable outcomes with combined direct and indirect revascularization in an even younger patient population form the basis of our recommendation to consider the direct technique.

\section{Limitations}

This study is a retrospective analysis of prospectively collected data. The database does not include pediatric patients treated conservatively during the study period, and does not include pediatric patients treated surgically by other neurosurgeons. This study was meant to demon- strate feasibility of direct bypass in this young age group, and did not aim to compare results with indirect bypass techniques.

\section{Conclusions}

The direct STA-MCA bypass procedure in combination with EMS for MMD is feasible and safe in patients 3 years or younger, based on favorable clinical and radiological outcomes in this cohort of patients. Direct bypass should be considered when immediate revascularization is needed, without the biological delay associated with indirect bypass.

\section{Acknowledgments}

Dr. Gyang Markus Bot is an AANS international visiting fellow for 2017 working under Dr. Michael T. Lawton.

\section{References}

1. Burke GM, Burke AM, Sherma AK, Hurley MC, Batjer HH, Bendok BR: Moyamoya disease: a summary. Neurosurg Focus 26(4):E11, 2009

2. Guzman R, Lee M, Achrol A, Bell-Stephens T, Kelly M, Do HM, et al: Clinical outcome after 450 revascularization procedures for moyamoya disease. Clinical article. J Neurosurg 111:927-935, 2009

3. Ikezaki K: Rational approach to treatment of moyamoya disease in childhood. J Child Neurol 15:350-356, 2000

4. Imaizumi T, Hayashi K, Saito K, Osawa M, Fukuyama Y: Long-term outcomes of pediatric moyamoya disease monitored to adulthood. Pediatr Neurol 18:321-325, 1998

5. Jackson EM, Lin N, Manjila S, Scott RM, Smith ER: Pial synangiosis in patients with moyamoya younger than 2 years of age. J Neurosurg Pediatr 13:420-425, 2014

6. Kim SK, Seol HJ, Cho BK, Hwang YS, Lee DS, Wang KC: Moyamoya disease among young patients: its aggressive clinical course and the role of active surgical treatment. Neurosurgery 54:840-846, 2004

7. Kim T, Oh CW, Bang JS, Kim JE, Cho WS: Moyamoya disease: treatment and outcomes. J Stroke 18:21-30, 2016

8. Kuroda S, Houkin K: Moyamoya disease: current concepts and future perspectives. Lancet Neurol 7:1056-1066, 2008

9. Matsushima T, Inoue T, Ikezaki K, Matsukado K, Natori Y, Inamura T, et al: Multiple combined indirect procedure for the surgical treatment of children with moyamoya disease. A comparison with single indirect anastomosis and direct anastomosis. Neurosurg Focus 5(5):e4, 1998

10. Rashad S, Fujimura M, Niizuma K, Endo H, Tominaga T: 
Long-term follow-up of pediatric moyamoya disease treated by combined direct-indirect revascularization surgery: single institute experience with surgical and perioperative management. Neurosurg Rev 39:615-623, 2016

11. Scott RM, Smith ER: Moyamoya disease and moyamoya syndrome. N Engl J Med 360:1226-1237, 2009

12. Smith ER, Scott RM: Surgical management of moyamoya syndrome. Skull Base 15:15-26, 2005

13. Thines L, Petyt G, Aguettaz P, Bodenant M, Himpens FX, Lenci H, et al: Surgical management of Moyamoya disease and syndrome: current concepts and personal experience. Rev Neurol (Paris) 171:31-44, 2015

14. Veeravagu A, Guzman R, Patil CG, Hou LC, Lee M, Steinberg GK: Moyamoya disease in pediatric patients: outcomes of neurosurgical interventions. Neurosurg Focus 24(2):E16, 2008

15. Wang KC, Phi JH, Lee JY, Kim SK, Cho BK: Indirect revascularization surgery for moyamoya disease in children and its special considerations. Korean J Pediatr 55:408-413, 2012

16. Yu J, Shi L, Guo Y, Xu B, Xu K: Progress on complications of direct bypass for moyamoya disease. Int J Med Sci 13:578-587, 2016

17. Zipfel GJ, Fox DJ Jr, Rivet DJ: Moyamoya disease in adults: the role of cerebral revascularization. Skull Base 15:27-41, 2005

\section{Disclosures}

The authors report no conflict of interest concerning the materials or methods used in this study or the findings specified in this paper.

\section{Author Contributions}

Conception and design: Lawton, Burkhardt, Gupta. Acquisition of data: Bot, Burkhardt. Analysis and interpretation of data: Lawton, Bot, Burkhardt. Drafting the article: Bot, Burkhardt. Critically revising the article: Lawton, Burkhardt. Reviewed submitted version of manuscript: all authors. Approved the final version of the manuscript on behalf of all authors: Lawton. Statistical analysis: Burkhardt. Study supervision: Lawton, Gupta.

\section{Supplemental Information}

\section{Current Affiliations}

Dr. Burkhardt: Department of Neurosurgery, Baylor College of Medicine Medical Center, Houston, TX.

\section{Correspondence}

Michael T. Lawton: Barrow Neurological Institute, Phoenix, AZ. michael.lawton@barrowbrainandspine.com. 